International Journal of Engineering \& Technology, $7(2.32)(2018) 209-211$
International Journal of Engineering \& Technology
SPC
Website: www.sciencepubco.com/index.php/IJET
Research paper

\title{
Accessing E-Commerce Through Social Media: Recommending Cold-Start Product Using Advanced Feature Mapping Method
}

\author{
Dr.T.Vijaya Saradhi ${ }^{1}$, V.Sai Vineetha ${ }^{2}$, J.Prathyusha ${ }^{3}$, K.Sai $^{\text {Teja }}{ }^{4}$ \\ ${ }^{1,2,3,4}$ Department of CSE, KL E F, Guntur, India. \\ *Corresponding author E-mail: saradhi1440@kluniversity.in
}

\begin{abstract}
As of late, the outskirts between electronic trade and informal organizations are deteriorating. Many e-commerce sites support social access mechanisms where users can log into sites utilizing their interpersonal organization accounts. Clients can also put their recently obtained products with correspondence to online business items on microblogs. In this article, we suggest another answer for a product of cold launch crossed, which aims to furnish clients of ecommerce sites with long range interpersonal communication locales in situations of "cold start" which have been studied very rarely. A great problem is how to gain influence through information long range interpersonal communication to offer cross-start basic products. We recommend that users use social networking websites and ecommerce websites as social networking capabilities on the map as a bridge on the map, another representation of product offerings. Specifically, we offer using user-generated and product-specific presentations of e-commerce sites that use regular neural networks and then use modified degraded trees to convert users' social network functions investments. We then develop a matrix factor approach based on features that can be used to invest in the cold start initiated by the user. The experimental results of China's largest microblogging service, SINAWEIBO and Jing Dong's biggest B2C ecommerce site have demonstrated the adequacy of the proposed scope.
\end{abstract}

Keywords:E-Commerce, product recommender, product demographic, microblogs, recurrent neural networks.

\section{Introduction}

As of late, the outskirts between electronic trade and informal organizations are deteriorating. On online business sites, for example, eBay, numerous long range informal communication highlights, including continous updates and associations among purchasers and merchants. Some web based business sites also support social access mechanisms that allow new clients to access their social network services for instance, Facebook and Twitter introduced another component a year prior that enables customers to buy their things straight forwardly from the sites by tapping on the purchase catch, advertisements or different items to purchase made a key sense of duty regarding SINA

WEIBO1, where ALIBABA item declarations can be conveyed specifically to SINA WEIBO customers. It is

important for the new direction of e-commerce in social networks develop product delivery systems to take advantage of the knowledge acquired in social networks. In this article, we analyze the products of e-commerce sites to offer users of social networks an interesting topic that has no historical purchases, that is, in situations of "cold start". We call this problem a cold-crossover product. Nevertheless the online product offering has been studied extensively, focusing mainly on research on the creation of ecommerce sites and, often, using historical transactional users. The best option for our knowledge, the cold-superior product proposal, has been rarely studied. Only users of social network information are used to solve our problem, and it is difficult to transform the data of informal communities into secret features that can be viably used for the item offers. To address this test, we urge clients to utilize person to person communication sites and web based business sites that have social networking records and shopping on ecommerce websites as an extension to delineate interpersonal interaction highlights. Product recommendation in particular, we offer you learn how to use products and product's feature presentations are referred to as user investment and item investments, separately, from information gathered from ecommerce websites utilized by general neural system and then using a payper-action variable tree method to transform social media capabilities of users in investments. Then we develop a matrix factorization approach based on features that can be used for client investment cold start product recommendation. We have manufactured our data collection of China's biggest microblogging service, SINAWEIBO2, and China's largest B2C ecommerce site, JINGDONG, which has a sum of 20,638 supporters. The exploratory consequences of information accumulation have demonstrated that the accommodation and adequacy of our proposed scope are as follows. Our main investments are summarized below. We are creating a novel problem from the ecommerce site, the products are recommended by users of social networks in situations of extreme cold. We recommend using the following neural network to teach users and actions related to the product for the use of information collected from the ecommerce website. We offer different graphic growth trees strategy to transform clients microblogging functions into a secret representation that can be effortlessly incorporated for recommending a item. We recommend a function based matrix factorization approach that uses the client and product properties for the cold start product recommendation. 


\section{Literature Survey}

We feature related critical methodologies: 1) customary synergistic recommender, 2) trust-improved recommender, and 3) audits based recommender. In the first place, the conventional cooperative sifting methodologies can be either memory-based or display based. These techniques depend on the rating history from clients. In the memorybased strategies, comparability calculation is an essential component. They utilize a heuristic utility of likeness between clients' vectors, for example, Pearson Correlation Coefficient (PCC) or cosine comparability measure (VCC) [1], [5], [13], [14]. Then again, the model-based strategies utilize machine learning models to anticipate item appraisals [15], [16]. For instance, Sarwar et al. [4], [17] actualized bunching calculations to recognize gatherings of clients who appraised comparative items and these groups can be viewed as likeminded neighbors. Since k groups are made, suggestion expectation can be figured by averaging the evaluations in that bunch. Miyahara and Pazzani [5] proposed a RS in view of Naive Bayes classifier and they just considered things which co-appraised between clients. They controlled two classes: like and don't care for and highlights are chosen in a preprocessing step. Late proposition centered in the precision of expectations, for example, lattice factorization for synergistic sifting. The approach proposed in [8] included social associations information in giving suggestion by relegating social regularization terms with a specific end goal to imperative grid factorization target work. They expected that companions rate items and consequently they utilized PCC and VCC to gauge similitude as moderate advance. Second, more investigations have concentrated on trust-improved recommenders. A few investigations connected trust by building trust net-work in view of the supposition that clients can acquire more exact proposal from individuals they trust [18], [19]. These sorts of techniques utilized direct assessments of trust from clients. Golbeck et al. [18] proliferated trust from trust arrange alleged Web Of Trust WOT. Just companions whose trust assessment surpasses a limit will be engaged with suggestion experience. Suggestions are gotten by weighted normal of evaluations alongside the trust esteem utilizing Film Trust dataset. In another specific circumstance, Massa et al. [19] utilized trust to channel the arrangement of neighbors and just their appraised things would be considered in foreseeing evaluations to a dynamic client. Subsequent to separating neighbors, they connected the customary proposal calculation. The examinations depended on Opinion dataset which contains the two clients' appraisals and the immediate trust esteems from clients towards each other. Third, late examines have been done to abuse the opinion in the printed audits to expand appraisals in collective recommenders [11], [12], [20]. Creators in [11] attempted to enhance the RSs by utilizing point and notion data at sentences level. They construed appraisals from content audits composed by clients about eateries in multipoint rating scale instead of just positive or negative polarities. They connected content relapse model to assess scaled slant appraisals. They are the primary who incorporated the helpful data in audits into RSs. Lenug et al. [12] proposed a probabilistic estimation deduction system. They connected characteristic dialect systems to process assumption introduction in audits. They fabricated their rating induction demonstrate in view of the Naive Bayes classifier. At that point, they incorporated between the deduction appraisals from surveys and a CF calculation to expand clients' inclinations and accomplished empowering comes about. Esparza et al. in [21] examined how to get suggestion from online smaller scale blogging administrations. They proposed an answer for misuse short posts composed by clients as item surveys. These presents are utilized on assemble client thing profile. At that point a question seek calculation is connected to recover pertinent thing profiles in view of a twitter-like survey benefit called blipper.com. This investigation is like our work in utilizing miniaturized scale blogging as a wellspring of proposal. Some inalienable downsides still have not been fathomed in the previously mentioned tech- niques. A large portion of these methodologies expect clients to deliver some organized information first, for example, confide in assessments and evaluations to enable the relating frameworks to work appropriately. Actually, this isn't commonsense and as a rule not accessible. All things considered, the shortcomings of sparsity and cool begin issues show up on account of trust arrange as it is in the client thing rating network. Then again, survey based recommenders require a client to compose audits and rate items to create the proposals. Dissimilar to existing investigations, our novel approach ISTS conquers the need of appraisals or composed surveys by clients and mirrors the genuine shrouded social put stock in relations. In our work we customize proposals from smaller scale loggings utilizing slant examination and trust between companions.

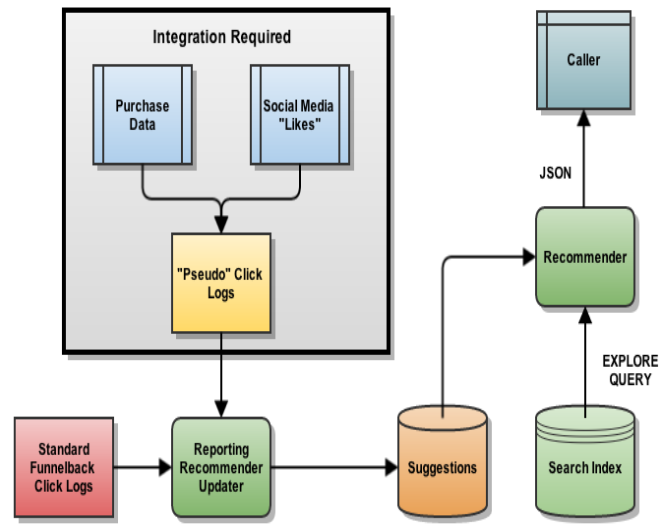

Fig1. System architecture for accessing ecommerce through social media

\section{Problem Formulation}

Given an online business site, Let U signify an arrangement of its customers, $\mathrm{P}$ a game plan of things and $\mathrm{R}$ purchase record system, every section of which is a twofold regard demonstrating whether the $U$ has obtained a thing P. Each and every customer $U$ is related with the course of action of obtained things with the purchase timestamps. Besides, a little subset of customers in $U$ can be associated with their microblogging accounts demonstrated. All things considered, every client $\mathrm{u}$ is connected with their individual microblogging characteristic data. Let A be denoted as the course of action of microblogging features and every microblogging customer has a jAj-dimensional microblogging feature vector au, in which every section au; I is the property estimation for the ith microblogging quality consolidate. We consider a cross site cold start situation: A microblogging client $\mathrm{u} 0=2 \mathrm{Uis}$ new to the online business webpage, who has no chronicled purchased records. It is anything but difficult to see $\mathrm{u} 0=2 \mathrm{UL}$, as well, since we have UL $\mathrm{U}$. We intend to create a customized positioning of suggested items for $\mathrm{u} 0$ in view of her microblogging properties au0. Because of the heterogeneous nature between these two unmistakable information signals, data expelled from microblogging organizations can't as a general rule be used particularly for thing proposition on online business locales. Along these lines, one noteworthy test is the means by which to change clients' microblogging quality data au0 into another component portrayal vu0, which can be utilized all the more successfully for item proposal. Here, we call au0 the first or microblogging highlight portrayal and vu0 the (heterogeneous) changed element representation, respectively. Secondly, we will contemplate how to remove microblogging highlights and change them into a passed on incorporate depiction before showing a component based system factorization approach, which merges the informed scattered component depictions for thing proposal. The entire work flow of our answer is appeared in which comprises of four imperative advances part into the feature mapping and item proposal, which will be talked about in Sections 3 and 4 independently. 


\subsection{Heterogenous Representation Mapping Using the Gradient Boosting Regression Trees Method:}

We acquainted how to develop a microblogging highlight vector au from a microblogging webpage and take in a dispersed depiction vu from a web based business site individually. In the cross webpage cold start proposal issue which we are considering in this paper (i.e., make a thing recommendation to customer $U$ who hasn't gained any items from the online business webpage), We can just get the microblogging feature vector au for customer $\mathrm{u}$ The key thought is to utilize few associated customers crosswise over locales as an extension to take in a capacity which maps the first element portrayal au to the dispersed portrayal vu. Basically, we have to develop a preparation set containing feature vector sets, and cast the component mapping issue as an administered relapse errand: the data is a microblogging feature vector au and yield is an appropriated incorporate vector vu. Accept that vu contains $\mathrm{K}$ measurements, we have to take in an arrangement of $\mathrm{K}$ capacities , and the ith work takes the principal segment vector of a customer $\mathrm{U}$ as the data and it restores relating to the changed element esteem, i.e., . We widen the Multiple Additive Regression Tree procedure to learn incorporate mapping limits since it is intense to get higher arrange change connection amongst data and yield.

\section{Experimental Results}
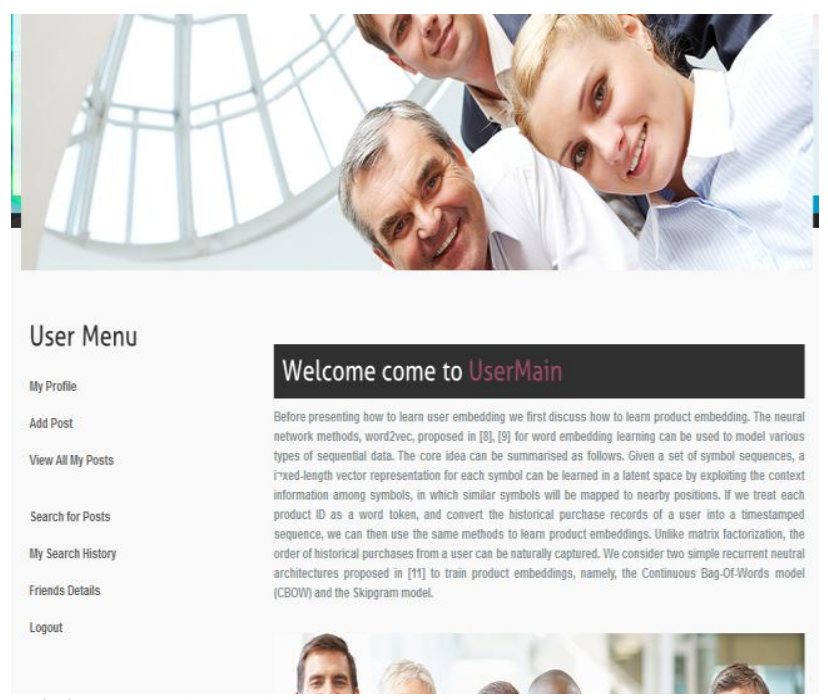

Fig2. Connecting social media to ecommerce to recommend a product

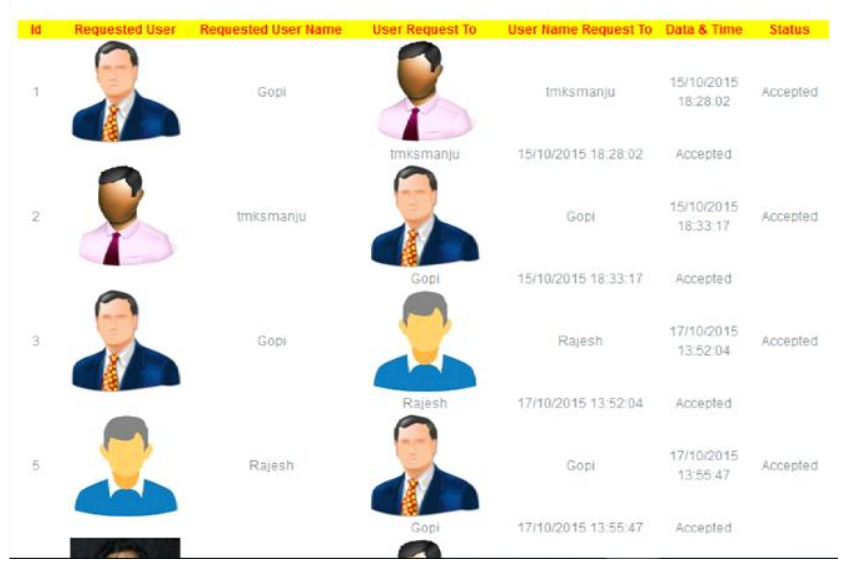

Fig 3. Recommendation of a product to a peer through social media

\section{Conclusion}

We have inspected a novel problem in this paper, cross-webpage cold start item recommendation, i.e., supporting things from the web business areas to microblogging customers without related buy records. Our significant notion is that on the internet based business areas, customers and items can be tended in the same torpid part space through component learning with the sporadic neural

systems. Utilizing stinations and long range casual correspondence regions as a framework, we can learn feature mapping limits utilizing a modified edge boosting trees technique, which maps clients properties removed from individual to singular correspondence objectives onto fuse delineations picked from internet based business areas. The mapped customer highlights can be effectively intertwined into a segment based system factorization approach for cold start thing suggestion. We have gathered a tremendous dataset from Jingdong and Weibo. The results display that our proposed framework is in actuality sensible to the cross site cold start thing suggestion issue. We assume that our analysis will have basic effect on both research and industry gatherings. Starting today, only a fundamental unprejudiced framework designing has been utilized for customer and thing embeddings learning. Later on, additionally created huge learning models, for instance, ConvolutionalNeuralNetworks can be explored for feature learning. We will like way consider enhancing the present Component Mapping Method through ideas in exchanging learning.

\section{References}

[1] J. Wang and Y. Zhang, "Opportunity model for e-commerce recommendation: Right product; right time," in SIGIR, 2013.

[2] M. Gyring, "Retail sales prediction and item recommendations using customer demographics at store level," SIGKDD Explor. Newsl., vol. 10, no. 2, Dec. 2008.

[3] G. Linden, B. Smith, and J. York, "Amazon.com recommenda-tions: Item-to-item collaborative filtering," IEEE Internet Computing, vol. 7, no. 1, Jan. 2003.

[4] V. A. Zeithaml, Sarwar et al "The new demographics and mar-ket fragmentation,” Journal of Marketing, vol. 49, pp. 64-75, 1985.

[5] W. X. Zhao, Y. Guo, Y. He, H. Jiang, Y. Wu, and X. Li, Mi-yahara and Pazzani "We know what you want to buy: a demograph-ic-based system for product recommendation on microblogs," in SIGKDD, 2014.

[6] J. Wang, W. X. Zhao, Y. He, and X. Li, "Leveraging product adopter information from online reviews for product recommendation," in ICWSM, 2015.

[7] Y. Seroussi, F. Bohnert, and I. Zukerman, "Personalised rating prediction for new users using latent factor models," in ACM HH, 2011.

[8] T. Mikolov, I. Sutskever, K. Chen, G. S. Corrado, and J. Dean, "Distributed representations of words and phrases and their compositionality," in NIPS, 2013.

[9] Q. V. Le and T. Mikolov, "Distributed representations of sen-tences and documents," CoRR, vol. abs/1405.4053, 2014.

[10] J. Lin, K. Sugiyama, M. Kan, and T. Chua, "Addressing cold-start in app recommendation: latent user models constructed from twitter followers," in SIGIR, 2013.

[11] T. Mikolov, K. Chen, G. Corrado, and J. Dean, "Efficient estimation of word representations in vector space," CoRR, vol. abs/1301.3781, 2013.

[12] RSs. Lenuget al. ,Y. Koren, R. Bell, and C. Volinsky, "Matrix factorization techniques for recommender systems," Computer, vol. 42, no. 8, pp. 30-37, Aug. 2009.

[13] J. H. Friedman, "Greedy function approximation: A gradient boosting machine," Annals of Statistics, vol. 29, pp. 1189-1232, 2000.

[14] L. Breiman, J. Friedman, R. Olshen, and C. Stone, Classifica-tion and Regression Trees. Monterey, CA: Wadsworth and Brooks, 1984.

[15] L. Breiman, "Random forests," Mach. Learn., vol. 45, no. 1, Oct. 2001.

[16] K. Zhou, S. Yang, and H. Zha, "Functional matrix factoriza-tions for cold-start recommendation," in SIGIR. 